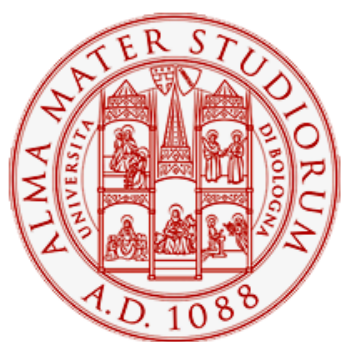

Alma Mater Studiorum - Università di Bologna DEPARTMENT OF ECONOMICS

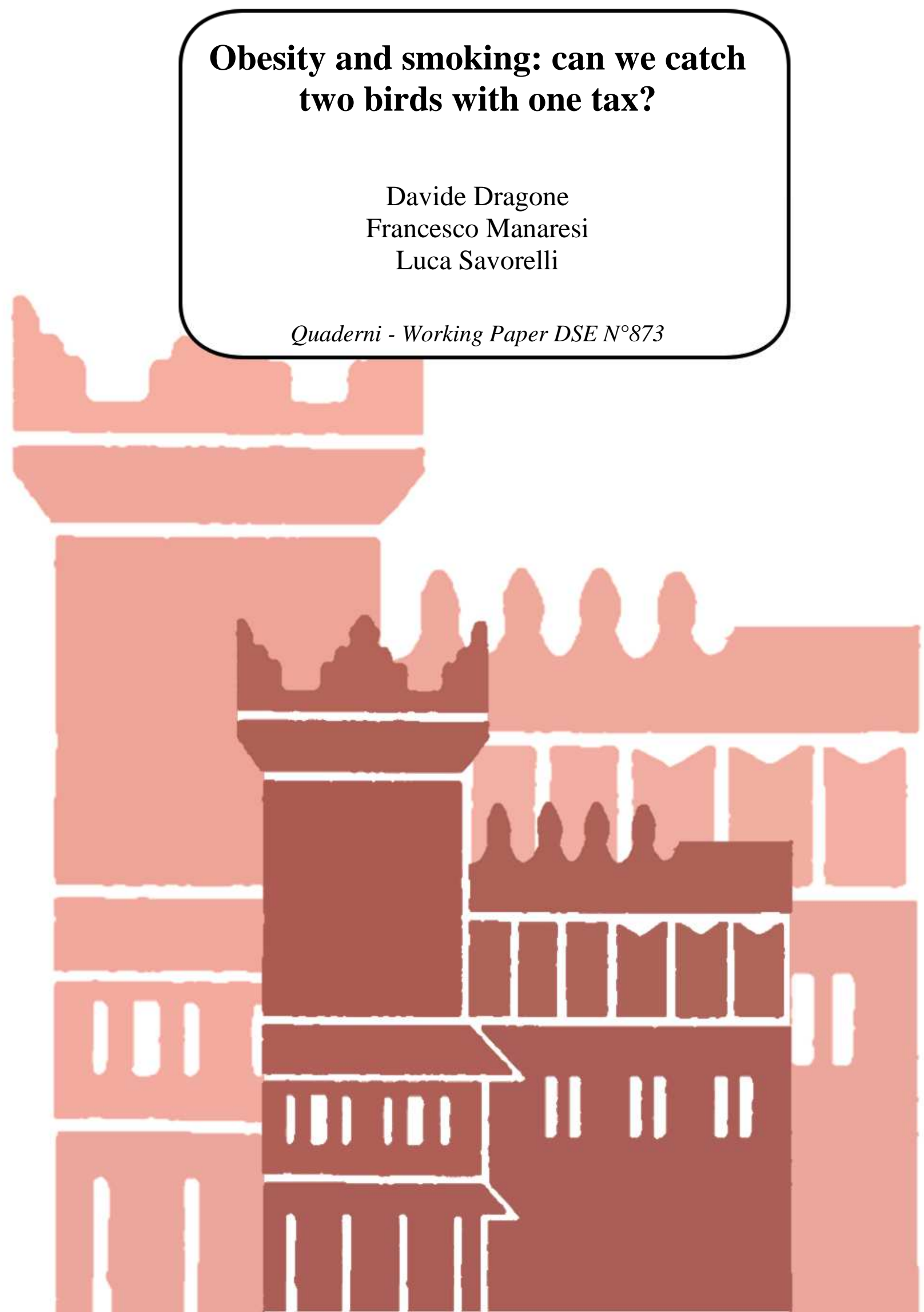




\title{
Obesity and smoking: can we catch two birds with one tax?*
}

\author{
Davide Dragone $^{\dagger} \quad$ Francesco Manaresi ${ }^{\ddagger} \quad$ Luca Savorelli $^{\S}$
}

March 26, 2013

\begin{abstract}
The debate on tobacco taxes and fat taxes often treats smoking and eating as independent behaviors. However, since there exists medical and sociological evidence about the interdependence between eating and smoking choices, antismoking policies may also affect the obesity prevalence and fat taxes could influence smoking behavior. We address this issue from a theoretical standpoint and propose a dynamic rational model where eating and smoking are simultaneous choices that jointly affect body weight and addiction to smoking. Focusing on direct and cross price effects, we compare tobacco taxes and fat taxes and we show that a single policy tool can reduce both smoking and body weight. In particular, fat taxes can be more effective than tobacco taxes at simultaneously fighting obesity and smoking.
\end{abstract}

Keywords: Addiction, Fat Tax, Obesity, Smoking, Tobacco.

JEL code: D91, H31, I18.

${ }^{*}$ We thank for useful discussion Giacomo Calzolari, Arsen Palestini, Giuseppe Pignataro, Giovanni Prarolo, Paolo Vanin and the participants in the ECHE 2012, PET 2012, 12th Viennese Workshop on Optimal Control, Dynamic Games and Nonlinear Dynamics, and in the seminars at the University of Alicante, Lausanne, Modena and Reggio Emilia, York, St. Andrews, Bologna. Financial support from FarmaFactoring Foundation and from the SoNIC project (FIRB grant no. RBFR084L83) is gratefully acknowledged. The views expressed in this paper are the authors' own and do not necessarily reflect those of the Bank of Italy. The usual disclaimer applies.

${ }^{\dagger}$ Corresponding author: Davide Dragone, University of Bologna, Dipartimento di Scienze Economiche, Piazza Scaravilli 1, 40126 Bologna, Italy; Phone: +39-051-209-8880, Fax: +39-051-209-8143, E-mail: davide.dragone@unibo.it.

${ }^{\ddagger}$ Bank of Italy, Structural Economic Analysis - Labour Market Division, via Nazionale 91, 00184 Rome, Italy; Phone:+39-06-479-224-26; E-mail: francesco.manaresi@esterni.bancaditalia.it.

${ }^{\S}$ University of St Andrews, School of Economics \& Finance, Castlecliffe, The Scores, St Andrews, Fife KY16 9AR, Scotland, United Kingdom. Phone: +44-1334-462449; e-mail: luca.savorelli@st-andrews.ac.uk. 


\section{Introduction}

In this paper we propose a dynamic model where eating and smoking are interdependent choices and we show under which conditions a single policy tool suffices to reduce both smoking and obesity. Comparing taxes on tobacco and taxes on food, we show that the latter can be more effective in simultaneously reducing obesity and smoking. This result has important policy implications, as smoking and obesity are two major causes of preventable death. In the United States, almost one out of four deaths is estimated to be due to tobacco smoking or obesity, which also have a significant impact on the health care system, with yearly medical expenses and related costs of about the magnitude of $\$ 117$ billions for smoking and $\$ 190$ billions for obesity (Cawley and Meyerhoefer, 2012; Mokdad et al., 2004). Since both produce externalities or, in case of time inconsistent choices, internalities (Chaloupka and Warner, 2000; Evans et al., 1999; Gruber, 2001; Gruber and Köszegi, 2001, 2004; Finkelstein et al., 2004), national governments have tried to modify smoking and eating behavior through the introduction of taxes, educational interventions, advertising campaigns, and the provision of assistance and tutoring.

The evaluation of the effectiveness of these policies and the debate about the introduction of new ones usually focus on their direct effects. For example, tobacco taxes are considered to be effective if smoking prevalence in the population decreases, and the debate on imposing taxes on energy-dense food (often called fat taxes) concentrates upon their expected effects on obesity rates. This approach can be appropriate when the indirect effects of the policy interventions are negligible. When considering smoking and obesity, however, this appears not to be the case, as suggested by the medical and sociological literature showing that smoking, eating behavior and body weight are interdependent and reciprocally influence each other. Interestingly, in the United States average smoking prevalence has been constantly decreasing in the last forty years (passing from $42 \%$ in 1965 to $20 \%$ in 2007, CDC, 2010) while, in the same span of time, obesity rate has increased (from $12.8 \%$ to over $30 \%$, Flegal et al., 1998, 2012). This evidence has stimulated a lively research agenda which studies whether the observed time-trends in smoking and obesity rates are the causal result of antismoking campaigns, rather than just a suggestive correlation. The economic literature on this issue is relatively recent and so far the empirical findings are mixed. In line with the common wisdom and the medical evidence that nicotine boosts metabolism and reduces appetite, some studies find that a raise in cigarette prices and in excises on tobacco increases average body mass index (BMI) in the population (Chou et al., 2004; Rashad and Grossman, 2004; Rashad et al., 2006; Baum, 2009). Other papers point to the opposite direction, finding that an increase in cigarette prices decreases BMI (Gruber and Frakes, 2006; Courtemanche, 2009; Wehby and Courtemanche, 2012; Dragone et al., 2013), which would instead support the conclusion that smoking and obesity are complements, rather than substitutes. 
To interpret the empirical results and to guide future policy interventions, a theoretical framework is needed; however, to the best of our knowledge, there is no theoretical model which treats smoking and eating behavior as joint interdependent choices. To address this issue, in this paper we propose a dynamic model for understanding how smoking and eating choices are jointly determined, and to provide novel testable predictions on the impact of taxation on smoking and obesity. We exploit the results from a body of recent medical and sociological literature, and we allow for two different pathways through which smoking, eating behavior and body weight are related: individual preferences and the effect of smoking on metabolism. The model is dynamic because smoking is addictive, and because body weight and addiction to smoking build up over time (Becker and Murphy, 1988; Levy, 2002; Dragone, 2009). This allows to construct a theoretical benchmark featuring both interdependence between goods consumed at different dates, as well as interdependence between different goods consumed at the same date.

Using this dynamic framework, we identify which behavior is consistent with a forwardlooking rational agent and show that the demand for smoking can be partially imputed to the demand for weight control. This result rationalizes the empirical finding that smoking initiation is sometimes driven by the desire to reduce appetite and to control body weight and, analogously, that fear of gaining body weight plays an important role in the decision to quit smoking (Moran et al., 2004; Cawley et al., 2004; Cawley and Scholder, 2013). We also show that a person can reach a healthy body weight and be not addicted to smoking, although this is only one of the possible outcomes of the model. In general, less healthy outcomes can emerge, including situations in which a person (optimally) smokes, is overweight and on a diet, or smokes, is underweight and binges. Finally, focusing on a permanent price increase we study the long-run demand for smoking, food consumption and body weight in order to understand the effect of policy actions that affect the price of smoking and of food.

By studying the demand for food in a dynamic framework where individuals are concerned about their body weight, we contribute to the debate on the introduction and effectiveness of taxes on targeted types of food. Supported by the United Nations and by the World Health Organization, some countries (notably, Denmark, Hungary, and France) have recently introduced some forms of food taxation, although with controversial outcomes (Mytton et al., 2012). Other countries, such as Ireland, Italy, Peru, the United Kingdom and the United States are actively considering whether introducing fat taxes to reduce obesity rates. Despite the fact that some policy actions have already been implemented, the theoretical literature on the impact of fat taxes on body weight is surprisingly thin. In a recent contribution, Yaniv et al. (2009) study how fat taxes and time constraints can affect obesity due to the simultaneous interdependence between healthy and junk food, and physical exercise. Here we consider a different perspective which focuses on the simultaneous interdependence between eating and smoking choices, as well as on the intertemporal effects of individual behavior 
on future utility and on the accumulation of body weight and addiction. To the best of our knowledge, such a dynamic theoretical framework is missing in the literature, and this paper is a first step to fill this gap.

By emphasizing the role of interdependencies between smoking and eating behavior, we identify the critical conditions that determine whether a tax-based policy intervention may produce undesirable trade-offs such as, e.g., a reduction in smoking prevalence but an increase in obesity. Importantly we highlight the possibility for the policy-maker to "catch two birds with one tax", i.e. the possibility for the policy maker to implement a single policy action which curbs both smoking and obesity. This result provides a rationale for those empirical papers emphasizing the role of antismoking policies in the reduction of obesity (Gruber and Frakes, 2006; Courtemanche, 2009; Wehby and Courtemanche, 2012; Dragone et al., 2013), and it opens the way to further research on policies that accomplish multiple goals with a single policy tool.

Finally, we show that smoking and eating behavior asymmetrically react to changes in prices. This is due to the fact that the metabolic effect of nicotine impacts on the accumulation of body weight, while food consumption does not affect the accumulation of addiction to smoking. This property implies that the policy implications of taxing tobacco and taxing food can be very different in terms of individual behavior and outcomes. More specifically, we find that fat taxes can be more effective than tobacco excises in simultaneously fighting obesity and smoking. The intuition for this result is that an increase in cigarette prices curbs tobacco consumption, but also reduces the "dieting" effects of nicotine. As a consequence, body weight decreases only if the reduction in smoking also leads to a strong reduction in food intake that more than compensates for a slower metabolism. By contrast, increasing the price of food reduces food consumption, and consequently body weight, which in turn makes smoking less desirable as a dieting device.

The paper is structured as follows. In the next section we review the evidence on the interdependence between smoking and eating behavior, and we present the theoretical literature on the taxation of addictive goods and on fat taxes. In section 3 we introduce the model and we solve it. In section 4 we study the short- and long-run demand functions for smoking and food consumption, and we compare the effects of taxing tobacco or food. Discussion and final considerations are contained in section 5 .

\section{Literature review}

Smoking and eating behavior can be interdependent through different avenues. A survey of the literature suggests that smoking may affect body weight through two major non mutually exclusive channels. The first one relies on the evidence that nicotine is an appetite suppressor through the central nervous system (Mineur et al., 2011). This anorexic effect of 
nicotine would suggest that smoking has a satiating effect on food consumption. This is not a general rule, however, because biological factors interact with individual preferences and social factors. Indeed, smokers tend to crave for smoking more when they are eating than in other situational correlates (Dunbar et al., 2010) and there exists a category of smokers that accounts for about one fourth of the market, the so-called social smokers, who smoke primarily in social contexts, such as in bars, parties, restaurants, celebrations and in presence of others (Debevec and Diamond, 2012; Schane et al., 2009). Contrary to the trend for regular smokers, the proportion of social smokers is rising. Analyzing data from the 19962001 Behavioral and Risk Factor Surveillance System, the U.S. Center for Disease Control finds significant increases in the proportion of nondaily smokers among current smokers in 38 states (CDC, 2003). Social smoking is particularly prevalent among young people. In a 2004 cross-sectional study of college students, Moran et al. (2004) classified 51\% of the sample of current smokers as social smokers. Moreover, heavy smokers are more likely to be obese. Despite the anorexic effect of nicotine, this evidence thus suggests that overall complementarity among smoking and eating behavior cannot be excluded (Chiolero et al., 2008), and that the reciprocal reinforcement between smoking and eating behavior is channelled through individual preferences, lifestyle choices and social factors such as peer-pressure and the conviviality dimension of smoking and eating.

The second channel of interdependence between smoking and eating behavior is based on the medical evidence that nicotine accelerates metabolism, which in turn increases caloric consumption (Chiolero et al., 2008; Mineur et al., 2011). Consistent with this result, a recurrent finding in the medical literature is that nicotine administration causes reduction in body weight, and that increased body weight results after cessation of administration (Donny et al., 2011). Interestingly, smoking is often reported to be used as a method for weight control: smoking initiation among teenagers is motivated by concerns on body weight (Moran et al., 2004) and, in a similar vein, some people refrain to quit smoking because they fear post-cessation weight gain (Spring et al., 2009; Cawley and Scholder, 2013). A recent study has shown that in the US $46 \%$ of girls and $30 \%$ of boys smoke to control weight and that the demand for smoking can be partially derived from the demand for weight control (Cawley and Scholder, 2013).

The above evidence and the negative correlation over time between smoking and obesity prevalence have inspired the conjecture that, due to the interdependencies between eating and smoking behavior, the obesity epidemic might have been fostered by antismoking policies. Whether this is empirically the case, however, is controversial. Focusing on the effect of increases in the prices of cigarettes, Chou et al. (2004), Rashad and Grossman (2004), Rashad et al. (2006), Baum (2009) show that quitting smoking is associated with increased body weight, while Gruber and Frakes (2006), Courtemanche (2009), Wehby and Courtemanche (2012) arrive at the opposite conclusion. Using detailed clinical data on individual health, 
smoking, and dietary habits, in a companion paper (Dragone et al., 2013) we show that increasing excise taxes on tobacco decreases body weight and caloric intake and improves the quality of eaten food.

The effect of price increases for addictive goods has been addressed from a theoretical stance since the seminal contribution by Becker and Murphy (1988). The main idea of rational addiction is that current preferences for an addictive good can depend on past consumption of that good. Becker and Murphy show that a decision-maker may rationally choose to become addicted by trading-off the intertemporal benefits and costs of consuming the addictive good, and that the demand for the addictive good decreases when its price increases. An important policy implication of Becker and Murphy's model is that, to reduce the consumption of the addictive good, one should increase its price. This prescription has received much attention both in the policy practice and in the literature, and there exists a large empirical consensus on the aggregate effectiveness of interventions based on increasing the price of addictive goods such as smoking, alcohol and heroin, among others. Similar theoretical predictions are obtained by Gruber and Köszegi (2001) in a rational addiction model with time-inconsistent agents, and by Gul and Pesendorfer (2007), who develop a model where consumption of the addictive good is a tempting choice that erodes self-control in future periods.

In the theoretical literature on obesity, the role of fat taxes on body weight has not been explicitly addressed, yet. An exception is in Yaniv et al. (2009), who adopt a static approach to show that fat taxes, while reducing junk-food consumption, may encourage the preparation of healthy meals. Since this requires time for cooking and shopping healthy-ingredients, and time is constrained, less time for physical activity remains available and an increase, rather than a decrease, in body weight can result. Even though the literature on fat taxes is scarce, there exists a small, but growing literature which models eating behavior and its dynamic effects on body weight. Rational eating models share many features of rational addiction models: consumers are forward-looking and maximize intertemporal utility taking into account that past choices affect current utility. In the context of eating behavior, food consumption choices affect future body weight, which in turn affects future utility because having an unhealthy body weight reduces the survival probability of the agent (Levy, 2002; Dragone, 2009) or determines health losses (Dragone and Savorelli, 2012). When trading off the current and future consequences of eating behavior, both being overweight and underweight can be rational outcomes, including apparently pathological situations where a person is underweight and yet is on a diet, or is overweight and binges (Dragone and Savorelli, 2012). In the next section we merge these literatures on rational addiction and rational eating considering eating and smoking as simultaneous and interdependent choices. 


\section{A model of rational smoking and eating}

Consider a representative agent whose utility function $U(s, c, q, a, w)$ depends on smoking $s$, food consumption $c$, a composite good $q$, past smoking experiences $a$, and body weight $w$. The utility function $U(\cdot)$ is continuously differentiable, jointly concave, and with negative second order derivatives $U_{c c}, U_{s s}, U_{w w}, U_{a a}$. In the rational addiction model by Becker and Murphy (1988), an addictive good features reinforcement between past and current consumption, and tolerance. Reinforcement implies that the marginal utility of consuming an addictive good increases with past consumption of that good. As a consequence of reinforcement, the more a person is addicted, the more she desires to consume the addictive good. Tolerance means that utility from a given amount of consumption is lower when past consumption is greater. Formally, the reinforcement property requires past smoking to increase the marginal utility of current smoking $\left(U_{s a}>0\right)$, and tolerance implies $U_{a} \leq 0$ for $a \geq 0$. Beside the interdependence in consumption of the same good at different dates, we are also willing to assume interdependence in consumption of two different goods at the same date. In particular, based on the evidence reviewed in the previous section, we allow for current smoking to affect the marginal utility of food consumption, as indicated by the mixed derivative $U_{c s}$. Moreover, we allow for smoking to accelerate metabolism (due to the metabolic effect of nicotine), which contributes to reduce the accumulation of body weight. Hence the evolution of body weight $w$ depends on past and current eating behavior, as well as on current smoking, so that $\dot{w}(t)=g(c(t), w(t), s(t))$ with $g_{c} \geq 0$ for $c(t) \geq 0, g_{w} \leq 0$ for $w(t) \geq 0$ and $g_{s} \geq 0$ for $s(t) \geq 0$ (subscripts denote partial derivatives). The variable $a$ representing past smoking experiences (addiction to smoking) evolves over time depending on current and past smoking choices, $\dot{a}(t)=f(s(t), a(t))$, with $f_{s} \geq 0$ for $s \geq 0$ and $f_{a} \leq 0$ for $a \geq 0$.

Given the initial body weight $w_{0}$, addiction to smoking $a_{0}$ and wealth $b_{0}$, the agent must choose the path of food consumption, smoking and consumption of the composite good that satisfies the following intertemporal problem:

$$
\begin{aligned}
\max _{s(t), c(t), q(t)} & \int_{0}^{T} e^{-\rho t}[U(c(t), s(t), w(t), a(t), q(t))] d t \\
\text { s.t. } \dot{a}(t) & =f(s(t), a(t)) \\
\dot{w}(t) & =g(c(t), w(t), s(t)) \\
\dot{b}(t) & =r b(t)+M-p^{c} c(t)-p^{s} s(t)-q(t) \\
a(0) & =a_{0}, w(0)=w_{0}, b(0)=b_{0} \\
c(t) & \geq 0, s(t) \geq 0, q(t) \geq 0 \\
w(t) & >0, a(t) \geq 0,
\end{aligned}
$$

where $\rho>0$ is the intertemporal discount rate, $r$ is the market interest rate, $M$ is the 
instantaneous wage of the agent and $b(t)$ is the available wealth. The terms $p^{c}$ and $p^{s}$ represent the market prices of food and smoking, while the price of the composite good is normalized to one.

In the remainder of the paper, we will make some simplifying assumptions which do not affect the main results, but make the exposition more tractable. We will focus on the case where no saving nor borrowing is possible, which allows replacing the dynamic budget constraint (4) with the static budget constraint $M=p^{c} c(t)+p^{s} s(t)+q(t)$, and we will consider the following quasi-linear specification for the utility function

$$
U(s(t), c(t), a(t), w(t))+q(t) .
$$

This assumption rules out the existence of Giffen goods and implies that the composite good is non satiating. As in the literature on rational addiction and on rational eating, we also assume that the evolution of addiction to smoking and body weight follows a linear dynamics:

$$
\begin{aligned}
& \dot{a}(t)=s(t)-\delta_{a} a(t), \\
& \dot{w}(t)=c(t)-\varepsilon s(t)-\delta_{w} w(t) .
\end{aligned}
$$

The depreciation parameters $\delta_{a}, \delta_{w} \in(0,1)$ are exogenous and represent the rate of decay of addiction to smoking and body weight respectively (Becker and Murphy, 1988; Levy, 2002; Dragone, 2009). For expositional convenience, we set $\delta_{a}=\delta_{w}=\delta$. The parameter $\varepsilon \geq 0$ represents the metabolic channel through which smoking affects body weight (Filozof et al., 2004; Chiolero et al., 2008).

Finally, we will assume that past smoking does not interact with the marginal utility of current food consumption $\left(U_{a c}=0\right)$, and that body weight does not interact with the marginal utility of current and past smoking $\left(U_{w s}=U_{w a}=0\right)$ nor with the marginal utility of current food consumption $\left(U_{c w}=0\right) \cdot 1 \cdot 1$

\subsection{The optimal solution}

To solve the forward-looking maximization problem, construct the following current-value Hamiltonian function (omitting the time index):

$$
H=U(c, s, w, a)+M-p^{c} c-p^{s} s+\mu(s-\delta a)+\lambda(c-\varepsilon s-\delta w),
$$

where $\mu$ and $\lambda$ are the costate variables associated to $a$ and $w$, respectively. They represent the shadow value of body weight and past smoking experiences, i.e. how much the value of

\footnotetext{
${ }^{1}$ The assumption $U_{c w}=0$ implies that food is not addictive, which allows focusing on a scenario where smoking is the only addictive good. For a different approach considering multiple addictive goods, see Dragone et al. (2012).
} 
the discounted optimal stream of utility changes when there is a marginal variation in body weight and past smoking experiences.

Given joint concavity, the following conditions, together with the appropriate transversality conditions and equations (6) and (7), are necessary and sufficient for an internal solution:

$$
\begin{aligned}
H_{c} & =0 \Leftrightarrow U_{c}-p^{c}=-\lambda \\
H_{s} & =0 \Leftrightarrow U_{s}-p^{s}=-\mu+\varepsilon \lambda \\
\dot{\mu} & =(\delta+\rho) \mu-U_{a} \\
\dot{\lambda} & =(\delta+\rho) \lambda-U_{w} .
\end{aligned}
$$

The first order conditions (8) and (9) simultaneously determine the optimal food consumption and smoking choices at each point in time, given the current level of addiction and body weight and their respective shadow values. Note that, in a dynamic framework, the optimal choice of food consumption and smoking in general does not correspond to the satiating choice in which $U_{c}=U_{s}=0$, nor to the solution of a boundedly rational agent that neglects how her current eating and smoking choices are going to affect her future utility ${ }^{2}$ This occurs because in a forward-looking framework the agent takes into account the shadow prices of addiction and body weight, and their evolution over time as a consequence of her smoking and eating behavior ${ }^{3}$ Accordingly, since food consumption only affects the determination of body weight, condition (8) only depends on $\lambda$. Since nicotine can affect body weight by accelerating the individual metabolism (as measured by $\varepsilon$ ), optimal smoking also depends on body weight. For this reason both costate variables, $\lambda$ and $\mu$, appear in (9).

\subsection{Long-run equilibrium}

To determine the long-run dynamic properties of the optimal choices of the agent, we focus on the steady state of the problem for $T \rightarrow \infty, 4$ Before describing the possible types of steady state that are consistent with the model, we introduce some terminology. We say the agent is overweight if $U_{w}<0$ (the agent would increase her utility by decreasing body weight) and, conversely, she is underweight if $U_{w}>0$ (she would increase utility by increasing body weight). Using the same logic, we say the agent is on a diet if $U_{c}>0$ and is binging if $U_{c}<0$; analogously the agent is smoking less than she would like if $U_{s}>0$, and is smoking more than she would like if $U_{s}<0$.

\footnotetext{
${ }^{2}$ The optimal solution for an agent with bounded rationality is reported in the Appendix

${ }^{3}$ On the contrary, for a boundedly rational agent the value of $\lambda$ and $\mu$ is zero at all $t$.

${ }^{4}$ For a similar approach see Becker and Murphy (1988), Dockner and Feichtinger (1991), Levy (2002).
} 
Proposition 1 In steady state the following conditions hold,

$$
\begin{aligned}
U_{w} & =(\delta+\rho)\left(p^{c}-U_{c}\right) \\
U_{a} & =(\delta+\rho)\left(p^{s}-U_{s}\right)+\varepsilon U_{w} \\
\delta w^{s s} & =c^{s s}-\varepsilon s^{s s} \\
\delta a^{s s} & =s^{s s}
\end{aligned}
$$

where the superscript ss denotes the steady state.

Proof. See the Appendix.

Equation (12) shows the existence of a trade-off between marginal utility of eating and marginal utility of body weight. Analogously, equation (13) shows the trade-off between the marginal utility of smoking, the marginal harm of the addiction to smoking and the marginal impact of smoking on body weight $5^{5}$ The steady state body weight increases with food consumption, and decreases with smoking (eq. 14), while steady state addiction to smoking tracks the changes in steady state smoking (eq. 15).

Can an agent rationally converge to a stable situation where she is not addicted to smoking and she has an optimal body weight $\left(U_{a}=U_{w}=0\right)$ ? Yes, this is possible, although this outcome can emerge only as a special case ${ }^{6}$ In general, the following four types of steady states associated to some addiction to smoking and to a non-optimal body weight can result:

(i) Being overweight and on a diet, and smoking more than the agent would like;

(ii) Being overweight and on a diet, and smoking less than the agent would like;

(iii) Being underweight and binging, and smoking less than the agent would like;

(iv) Being underweight and on a diet, and smoking less than the agent would like.

Outcome (i) can occur only when smoking has a metabolic effect on body weight $(\varepsilon>$ $0)$. In such a case, smoking effectively acts as a dieting device which the agent uses to reduce body weight. In particular, this outcome describes an overweight agent who is already dieting, and that optimally smokes beyond satiation to try to maintain body weight under control. This theoretical result is consistent with the evidence of those people who are overweight and declare they initiated smoking, even if they recognized it is harmful, because their dieting efforts were not successful enough to reduce their body weight (Cawley et al.,

\footnotetext{
${ }^{5}$ Alternatively, equation 12 can be expressed as a relation between the marginal utility of body weight and the corresponding shadow value: $U_{w}=(\delta+\rho) \lambda$. Hence, when an individual is overweight, in steady state the shadow price $\lambda$ is negative, and when she is underweight it is positive. Also equation (13) can be expressed as a relation between the marginal harm of addiction to smoking, the shadow value of addiction to smoking $\mu$ and the metabolic effect of smoking on body weight $\lambda, U_{a}=(\delta+\rho)(\lambda-\varepsilon \mu)$.

${ }^{6}$ If $U_{w}=U_{a}=0$ when $\dot{c}=\dot{s}=\dot{w}=\dot{a}=0$, conditions 12 and 13 imply that, in steady state, $U_{c} / U_{s}=p^{c} / p^{s}$ holds. Interestingly, this is the same condition characterizing the familiar static optimizing condition under budget constraint. The reason is that, in this specific steady state, the shadow value of addiction to smoking and body weight is nil (see 10 and 11 .
} 
2004). Outcomes (ii) and (iii) describe the cases where an overweight agent stays on a diet to avoid getting even more overweight (Levy, 2002, Dragone, 2009) or where an underweight agent eats beyond satiation to avoid getting even more underweight (Dragone and Savorelli, 2012). Outcome (iv) describes the case where being on a diet despite being underweight is a long-run equilibrium. Dragone and Savorelli (2012) show that such an outcome can possibly occur in a scenario where there is social pressure to be thin. Here, instead, the result is due to the fact that, if the price of food is high, the agent optimally substitutes the composite good for food, and possibly ends up eating below the satiation level even if she is underweight. Finally, note that smoking more than one would like is optimal only for an overweight agent, as she would otherwise refrain to smoke up to satiation because addiction to smoking is harmful (eq. 13).

\section{The effect of prices on individual behavior}

In this section we study the effect of prices on food consumption, body weight and smoking. We first consider the effect of an increase in the price of smoking and the effect of an increase in the price of food; then we use the results of the analysis to study the effectiveness of policy actions aimed at directly affecting the cost of smoking (through, e.g. the introduction of excise taxes on tobacco), or the cost of eating food (through, e.g. the introduction of taxes on junk food).

For both cases we will determine short- and long-run effects. Short-run effects are computed considering body weight and past addiction, and the corresponding shadow values, as fixed. For the long-run price effects, we will focus on stable steady states.7 The results will depend on the degree of interdependence among variables in the utility function and on the dynamic evolution of the state variables. In particular, on the basis of the evidence reviewed in the previous section, the demand responses will depend on three main factors. The first one is the simultaneous interdependence between current food consumption and current smoking. On the one hand, there is medical evidence showing that nicotine is an appetite suppressor. On the other hand, sociological and psychological evidence suggests that, depending on individual lifestyles, situational cues and peer effects, eating can increase the desirability of smoking. When the first effect is dominant, we say that smoking has a satiating effect on eating. Formally, this case is represented by $U_{c s}$ being negative, i.e. the marginal utility of food consumption decreases when smoking increases (and, conversely, the marginal utility of food consumption increases when smoking decreases). When the second effect is dominant, smoking has a reinforcing effect on eating. Formally, this case corresponds to $U_{c s}$ being positive. The second factor concerns the intertemporal dependence between past and current smoking. Following the literature on rational addiction, we allow only for the

\footnotetext{
${ }^{7}$ The conditions characterizing stable steady states are reported in the Appendix.
} 
case where past smoking has a reinforcing effect on current smoking $\left(U_{s a}>0\right)$. The third factor concerns the metabolic effect of current smoking on body weight $(\varepsilon \geq 0)$. This form of interaction does not directly affect preferences, as it is a pure dynamic effect that affects the evolution of body weight. Hence, it is not taken into account by an agent that neglects the future impact of her current choices, but it will play a major role for a forward looking agent.

\subsection{Increasing the price of smoking}

We first focus on the direct effect of an increase in the price of smoking. The following applies:

Proposition 2 When the price of smoking increases, smoking decreases both in the shortand in the long-run.

Proof. See the Appendix.

The result above replicates a main finding in Becker and Murphy (1988): addicts do respond to incentives, and they reduce the consumption of the addictive good when it becomes more expensive, both in the short- and long-run. Novel results emerge when considering the effects of an increase in the price of smoking on food consumption and body weight.

Proposition 3 When the price of smoking increases:

1. In the short-run food consumption decreases if $U_{c s}>0$, and increases otherwise;

2. In the long-run

- Food consumption decreases if $U_{c s}>\sigma_{1}$, and increases otherwise;

- Body weight decreases if $U_{c s}>\sigma_{2}$, and increases otherwise;

where $\sigma_{1}=\varepsilon U_{w w} /[\delta(\delta+\rho)]$ and $\sigma_{2}=-\varepsilon U_{c c} /(\delta+\rho)$.

Proof. See the Appendix.

The instantaneous reaction of food consumption to the increased price of smoking only depends on the interdependence between current smoking and eating. Accordingly, smoking and food consumption are complements in the short-run if smoking reinforces eating; and they are substitutes in the short-run if smoking has a satiating effect on food consumption 8 In the long-run the picture becomes more complex, as the role of smoking on metabolism, of time preferences and of the rate of decay of addiction to smoking and body weight play a critical role. In particular, three cases can occur (see Figure 1).

\footnotetext{
${ }^{8}$ Recall that only substitution effects are at work because, due to the quasi-linear utility specification, all income effects are captured by changes in the demand for the composite good.
} 


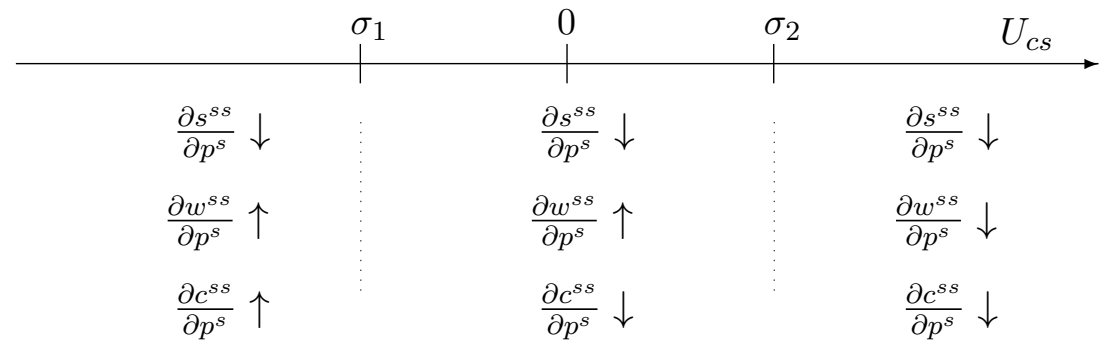

Figure 1: Long run effects of an increase in the price of smoking on smoking, body weight and food consumption.

When the reinforcing effect of smoking on food consumption is sufficiently strong $\left(U_{c s}>\right.$ $\sigma_{2}$ ), increasing the price of smoking reduces smoking, food consumption and body weight. In other words, smoking, eating and body weight are complements in the long-run. This outcome is consistent with recent empirical research suggesting that taxes on smoking have likely determined a decrease in body weight (Courtemanche, 2009; Wehby and Courtemanche, 2012) and in food intake (Dragone et al., 2013). Accordingly, antismoking policies not only have reduced smoking prevalence in the population, but also obesity. If, instead, the satiating effect of smoking is large enough $\left(U_{c s}<\sigma_{1}\right)$, food consumption and body weight are substitutes for smoking in the long-run. This case requires a careful assessment by the policy maker, because it preludes to the possibility that an antismoking policy, although effective in reducing smoking, increases food consumption and body weight. In the intermediate case where the intensity of the interdependence between current smoking and eating is mild $\left(\sigma_{1}<U_{c s}<\sigma_{2}\right)$, antismoking policies may foster the obesity epidemic even if people reduce their food intake. This result seems to fit the evidence of people who respond to antismoking policies by reducing smoking and food consumption, and yet increase their body weight. The reason hinges on the fact that smoking "burns calories" by accelerating individual metabolism. By reducing smoking, individual metabolism gets slower, which favours body weight accumulation. To compensate for the slower metabolism and the increased body weight, food intake is reduced. When the interdependence between smoking and food is mild (and possibly nil), however, the reduction in food intake is not enough to compensate the metabolic effect of reduced smoking, and body weight ultimately increases.

In the special case in which nicotine has no metabolic effect on body weight $(\varepsilon=0)$, then $\sigma_{1}=\sigma_{2}=0$, in which case the sign of the long-run reaction replicates the sign of the shortrun reaction and information on the interdependence between current smoking and eating is sufficient to predict the effect of an increase in the price of smoking on food consumption and body weight. 


\subsection{Increasing the price of food}

In this section we consider the effect of increasing the price of food on smoking, eating behaviour and body weight. The following proposition confirms that the law of demand holds also for food consumption.

Proposition 4 When the price of food increases, food consumption decreases both in the short- and in the long-run.

Proof. See the Appendix.

When considering the cross price effects, the short- and long-run reaction of smoking to changes in the price of consumption mirrors the reactions of food consumption to changes in the price of smoking. Interestingly, as shown in the Proposition below, the response of body weight to the price of smoking is different from the response to the price of food, due to the fact that smoking accelerates individual metabolism and that smoking is addictive.

Proposition 5 When the price of food increases:

1. In the short-run smoking decreases if $U_{c s}>0$, and increases otherwise;

2. In the long-run

- Smoking decreases if $U_{c s}>\sigma_{1}$, and increases otherwise;

- Body weight decreases if $U_{c s}<\sigma_{3}$, and increases otherwise;

where $\sigma_{3}=-\left[(2 \delta+\rho) U_{s a}+U_{a a}+\delta(\delta+\rho) U_{s s}\right] /[\delta(\delta+\rho) \varepsilon]$.

Proof. See the Appendix. 


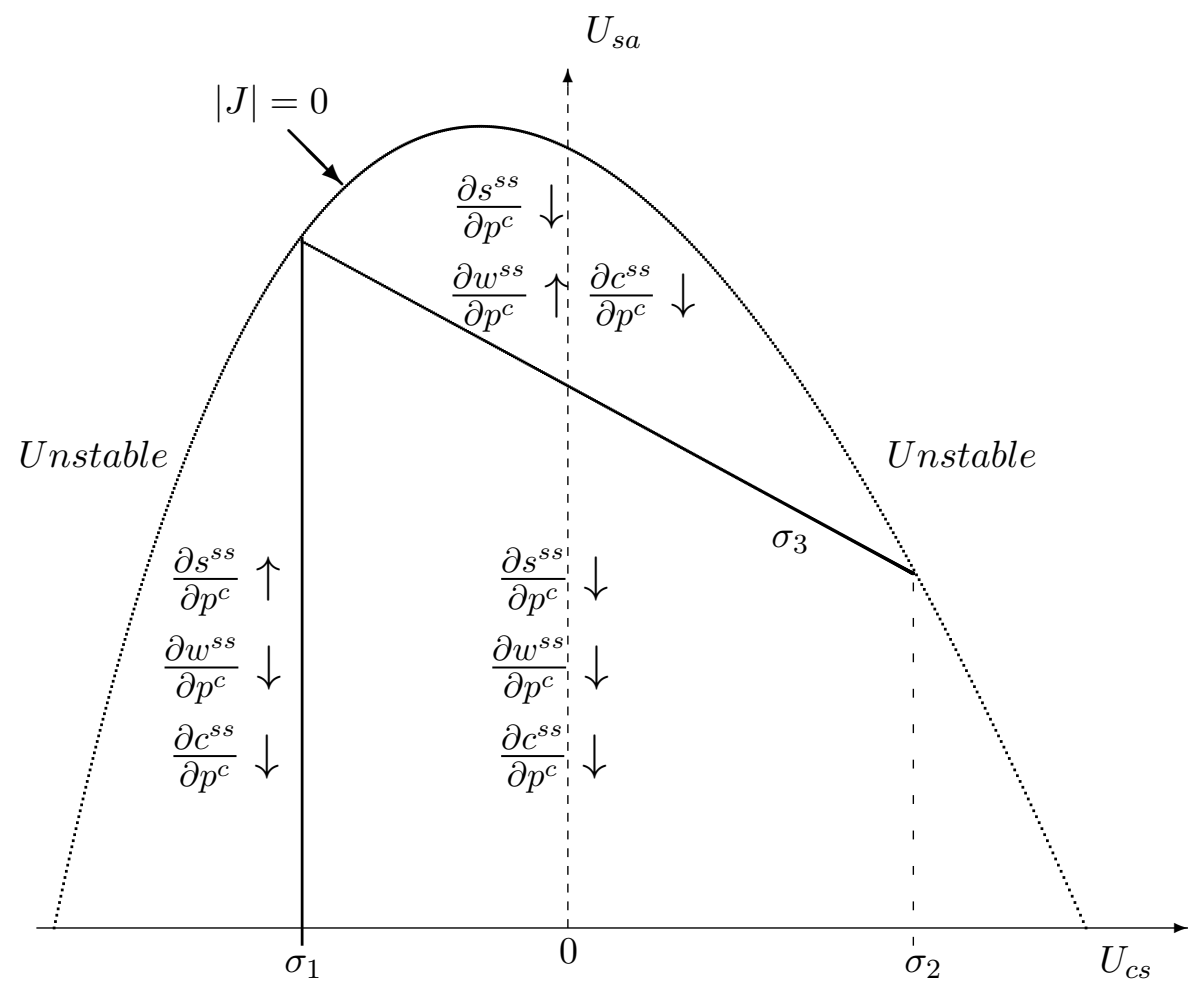

Figure 2: Long run effects of an increase in the price of food on smoking, body weight and food consumption.

Consider Figure 2, where we represent the cases described by the above Proposition 9 When the satiating effect of smoking on food consumption is large enough $\left(U_{c s}<\sigma_{1}\right)$, food consumption and body weight are complements in the long-run, while food consumption and smoking are substitutes. Hence in such a case the increase in the price of food is effective in reducing obesity, but the agent optimally substitutes smoking for food, which implies that the policy maker must carefully assess the trade-off between increased smoking and reduced obesity. In the area on the right of the vertical line indicated by $\sigma_{1}$, the reduction in food intake is accompanied by a reduction in smoking. The intuitive reason is that the fat tax directly discourages eating, which helps controlling body weight accumulation and reduces the incentives to smoke for losing weight. The intensity of the reduction in smoking depends on the intensity of $U_{s a}$ : the higher the reinforcing effect of past smoking on current smoking, the higher the reduction in current smoking after an increase in the price of food. In the area above the $\sigma_{3}$ line, the reduction in smoking is large, and the consequent slowing down of metabolism offsets the reduction in food intake. Hence, after an increase in the price

\footnotetext{
${ }^{9}$ In Figure 2 we consider the case where stability of the steady state requires $|J|>0$ and where all thresholds $\sigma_{1}, \sigma_{2}$ and $\sigma_{3}$ are feasible.
} 
of food, the agent reduces food intake and smoking, and yet her body weight increases. The area below the $\sigma_{3}$ line, instead, denotes the case where smoking is not very addictive. Consequently, although smoking is reduced, the effect on individual metabolism is dominated by the reduced food intake. This implies that, after an increase in the price of food, the agent will reduce eating, she will get thinner and she will also reduce smoking.

The latter result is interesting for two reasons. First, it highlights a possibility that does not appear to have been fully considered in the debate on eating policies against obesity, namely the possibility of implementing eating policies that contribute to reduce the smoking prevalence. In particular, this result is most relevant for those people who initiate and continue smoking because they are concerned about being overweight, in which case implementing policies that directly impact on food consumption and body weight would also make smoking less attractive. Second, there is an important asymmetry between the response of body weight to an increase in the price of smoking and an increase in the price of food consumption. While in both cases there is the possibility that smoking, food consumption and body weight are complements, when considering the price of food this outcome can occur in a larger set of cases: when smoking reinforces food consumption, when it has a mild satiating effect, and even when preferences for current smoking are independent from current eating. Although it is questionable whether focusing only on body weight and BMI provides enough information on the health condition of the agent, policy makers and institutions such as the World Health Organization are explicitly concerned about fat accumulation as a risk to health, and the corresponding campaigns are aimed at reducing obesity. Hence, identifying different effects on obesity prevalence as a response to different policy tools is of particular interest because it opens to the possibility of comparing the effectiveness of antismoking and antiobesity policies, as we do in next section.

\subsection{Taxing tobacco or taxing junk food?}

In this section we exploit the previously obtained results to compare the effectiveness of antismoking and antiobesity interventions. Our interest is on those policies that either raise the monetary cost of smoking (such as the introduction of excise taxes on tobacco) or the cost of food, everything else equal. Interpreting food consumption as a basket of different types of food, the latter case includes scenarios where taxes are imposed only on specific foods, as it is the case of fat taxes on junk food (energy-dense food, soft drinks etc.), raising the overall price of the basket. Based on the results contained in Propositions 2, 3, 4 and 5, the following holds:

Proposition 6 Fat taxes can be more effective than tobacco taxes to simultaneously fight obesity and smoking. 
The above Proposition highlights the fact that, although it is possible for the policy maker to reduce both obesity and smoking by using either tobacco taxes or fat taxes (provided there exists some interdependence among smoking and food consumption), their effect on body weight is not symmetric. By increasing the price of food, body weight and smoking decrease when current smoking and eating are either reinforcing or independent, or even when there is a mild satiating effect of smoking on eating (graphically, these cases correspond to the area on the right of $\sigma_{1}$ and below the $\sigma_{3}$ line, see Figure 2). When increasing the price of smoking, the same result can be obtained only if smoking is sufficiently reinforcing (area on the right of the $\sigma_{2}$ line). The result in Proposition 6 rests on the observation that the former region includes the latter one, which implies that the set of cases in which fat taxes determine a reduction in both smoking and obesity is (weakly) larger than the set where tobacco taxes accomplish the same goals.

The possibility of identifying a policy tool that reduces long-run obesity and smoking in a large range of cases is a relevant result because both smoking and obesity are major issues in the agenda of health authorities, due to the associated health risks (see, for example, WHO, 2000), to the production of externalities (both in terms of health, and in terms of hospitalization costs and medical care), and to the possible existence of internalities created by self-control problems. Since implementing a policy intervention is costly, showing that there exist differences in the effectiveness of policy actions provides information on which intervention is more desirable. In particular, the role of a dynamic framework must be emphasized, as the relative advantages of the two tools emerge from explicit consideration of the interdependence between different goods consumed at the same date, of the interdependence between the same good consumed at different dates, and on the interdependence in the evolution of body weight. In this direction, the above result allows to understand the variables that crucially determine the conditions under which no trade-off between smoking and obesity emerges, and it may provide a useful tool for guiding future policy interventions. 


\section{Conclusions}

In this paper we have introduced a dynamic model to jointly study the interaction between smoking and eating behavior over time, and to understand the conditions under which taxation aimed at affecting one health-related behavior may have a different scope than expected. We exploited the available medical and sociological evidence to support the assumption that smoking and eating behaviors are interdependent, either in terms of preferences or because they affect individual metabolism, and we have shown the trade-offs that characterize the rational behavior of a forward-looking agent. Consistent with the empirical evidence, we have found that a variety of stable eating and smoking outcomes may emerge, including clusters of unhealthy behaviors leading to being overweight and addicted to smoking, or being underweight and on a diet.

By emphasizing the role of interdependencies between different health-related behaviors, we show that targeting single health-related behaviors as if there were no interdependence with other behaviors can have a different scope than expected. This, however, is not necessarily bad news. Although it is possible for antismoking policies (or antiobesity policies) to backfire, there are conditions under which a single policy tool, such as the introduction of excises on tobacco, suffices to induce an overall health improvement by jointly reducing smoking and obesity rates in the population. In the latter case, the policy maker hits "two birds with one stone". We further studied whether taxing food provides any comparative advantage with respect to taxing cigarettes. We indeed found that the set of cases in which this two-for-one result is obtainable is larger when increasing the price of food than when increasing the price of smoking. This suggests that taxation of junk food may jointly reduce smoking and obesity prevalence more effectively than tobacco taxation. The intuition for this result is that a decrease in body weight also makes smoking less valuable as a dieting device, while a decrease in smoking slows down metabolism and reduces the efficiency of calorie burning.

Nevertheless, our results should be cautiously considered by the policy maker. First, our model may be interpreted as considering a representative agent, but distributional aspects of the population could play a role. The strength of reinforcement for smoking and the degree of interdependence between smoking and eating in preferences may substantially vary across socio-demographic groups. For example, the category of social smokers, which accounts for one fourth of the smoking market, is characterized by a mild addiction to smoking and a reinforcing effect between concurrent smoking and eating. In this case, our results predict that food taxation can be effective at reducing both smoking and body weight, while tobacco taxes may have an adverse effect on body weight. The policy maker could then introduce

food taxation only in specific places where the conviviality dimension is more relevant for social smokers (e.g. bars, restaurants, pubs). Second, food taxes and cigarette taxes are 
both regressive (Philipson and Posner, 2008, Gospodinov and Irvine, 2009, Haavio and Kotakorpi, 2011). We have not discussed here the regressive nature of taxation, nor provided a welfare assessment of the policies. In this respect, the policy maker should take into account the underlying asymmetry between smoking and eating behavior. Food taxation will impact the whole population, including thus normal weight individuals, smokers and non-smokers. Cigarette taxes are effective at curbing smoking consumption among smokers and discouraging smoking initiation, but do not impact non-smokers decisions on eating behavior and may have positive effects on body weight. Our cautionary message is thus that cigarette taxes should not be waived or reduced a priori and that the possibility to address two risk factors with food taxation should be balanced with the greater regressive effects that it may have.

The goal of this paper was to study the interaction between smoking, eating behavior and body weight over time, and to understand the conditions under which policies aimed at affecting one health-related behavior may have a different scope than expected. However, future research could extend this model by introducing choices over physical exercise, junk and healthy food, addictiveness of food, availability of information, self-control and timeinconsistency issues, and explicitly modelling social interactions.

Finally, the model we have proposed may be a useful starting point for research on other interrelated behaviors, which have already been subject of empirical analysis, e.g. use of multiple drugs, alcohol and tobacco (Bask and Melkersson, 2004), beer and marijuana (Pacula, 1998). The model can also be broadly applied in settings where the assumption of intertemporal separability of preferences is too restrictive, as it is the case of behaviors related to addiction, habit formation, learning-by-doing, and human capital formation.

\section{References}

Baum, C. L. (2009). The effects of cigarette costs on BMI and obesity. Health Economics, 18(1), 3-19.

Bask, M., Melkersson, M. (2004). Rationally addicted to drinking and smoking? Applied Economics, 36(4), 373-381.

Becker, G. S., Murphy, K.M. (1988). A theory of rational addiction. Journal of Political Economy, 96 (4), 675-700.

Cawley, J., Meyerhoefer, C. (2012). The medical care costs of obesity: an instrumental variables approach. The Journal of Health Economics, 31, 219 - 230.

Cawley, J., Scholder, S.H.K. (2013). The Demand for Cigarettes as Derived from the Demand for Weight Control, NBER working paper No. 18805.

Cawley, J., Markowitz, S., Tauras, J. (2004). Lighting up and slimming down: the effects of body weight and cigarette prices on adolescent smoking initiation. Journal of Health 
Economics, 23, 293-311.

CDC (2003). Prevalence of Current Cigarette Smoking among Adults and Changes in Prevalence of Current and Some Day Smoking-United States, 1996-2001. Morbidity and Mortality Weekly Report, 52(14), 303-07.

CDC (2009). State-specific Smoking-attributable Mortality and Years of Potential Life Lost- United States, 2000-2004. Morbidity and Mortality Weekly Report, 58(02), 29-33.

Chaloupka, F. J., Warner, K. E. (2000). The Economics of Smoking, in Handbook of Health Economics, 1, 1539-1627. Elsevier.

Chiolero, A., Faeh, D., Paccaud, F., Cornuz, J. (2008). Consequences of smoking for body weight, body fat distribution, and insulin resistance. The American Journal of Clinical Nutrition, 87(4), 801-809.

Chou, S., M. Grossman, H. Saffer (2004). An economic analysis of adult obesity: results from the Behavioral Risk Factor Surveillance System. Journal of Health Economics, 23, 565-587.

Courtemanche, C. (2009) Rising cigarette prices and rising obesity: Coincidence or unintended consequence? Journal of Health Economics, 28, 781-798.

Debevec, K., Diamond, W. D. (2012). Social smokers: Smoking motivations, behavior, vulnerability, and responses to antismoking advertising. Journal of Consumer Behaviour, 11(3), 207-216.

Dockner, E. J., Feichtinger, G. (1993). Cyclical consumption patterns and rational addiction. The American Economic Review, 83(1), 256-263.

Donny, E. C., Caggiula, A. R., Weaver, M. T., Levin, M. E., Sved, A.F. (2011). The reinforcement-enhancing effects of nicotine: implications for the relationship between smoking, eating and weight. Physiology \& behavior, 104(1), 143-148.

Dragone, D. (2009). A rational eating model of binges, diets and obesity. Journal of Health Economics, 28, 799-804.

Dragone, D., Manaresi, F., Savorelli, L. (2012). Multiple rational addictions and the effect of price on consumption, FarmaFactoring Foundation Working Papers.

Dragone, D., Manaresi, F., Savorelli, L. (2013). Tobacco taxes and smoking bans impact differently on obesity and eating habits, FarmaFactoring Foundation Working Papers.

Dragone, D., Savorelli, L. (2012). Thinness and obesity: a model of food consumption, health concerns, and social pressure. Journal of Health Economics, 31, 243-256.

Dunbar, M. S., Scharf, D., Kirchner, T. and Shiffman, S. (2010). Do smokers crave cigarettes in some smoking situations more than others? Situational correlates of craving when smoking. Nicotine and Tobacco Research, 12 (3), 226-234.

Evans, W., Farelly, M., Montgomery, E. (1999). Do workplace smoking bans reduce smoking? American Economic Review, 89, 728-747.

Filozof, C., Pinilla, F., Fernandez-Cruz, A. (2004). Smoking cessation and weight gain. 
Obesity Reviews, 5(2), 95-103.

Finkelstein, E., Fiebelkorn, I., Wang, G. (2004). State levels estimates of annual medical expenditures attributable to obesity. Obesity Research, 12, 18-24.

Flegal, K. M., Carroll, M. D., Kuczmarski, R. J. and others (1998). Overweight and obesity in the United States: prevalence and trends, 1960-1994. International journal of obesity and related metabolic disorders. Journal of the International Association for the Study of Obesity, 22, 39-47.

Flegal, K. M., Carroll, M. D., Ogden, C. L., Curtin, L. R. (2010). Prevalence and trends in obesity among US adults, 1999-2008. JAMA: the journal of the American Medical Association, 303 (3), 235-241.

Gospodinov, N., Irvine, I. (2009). Tobacco taxes and regressivity.Journal of health economics, 28(2), 375-384.

Gruber, J. (2001). Tobacco at the crossroads: The past and future of smoking regulation in the U.S. Journal of Economic Perspectives, 15(2), 193-212.

Gruber, J., Frakes, M. (2006). Does falling smoking lead to rising obesity? Journal of Health Economics, 25, 183-197.

Gruber, J., Köszegi, B. (2001). Is addiction 'rational?' Theory and Evidence. Quarterly Journal of Economics, 116(4), 1261-1305.

Gruber, J., Kőszegi, B. (2004). A theory of government regulation of addictive bads: optimal tax levels and tax incidence for cigarette taxation. Journal of Public Economics, 88 (9-10), 1959-1987.

Gul, F., Pesendorfer, W. (2007). Harmful addiction. The Review of Economic Studies. $74,147-172$.

Haavio, M., Kotakorpi, K. (2011). The political economy of sin taxes. European Economic Review, 55(4), 575-594.

Levy, A. (2002). Rational eating: can it lead to overweightness or underweightness? Journal of Health Economics, 21, 887-899.

Mineur, Y.S., Abizaid, A., Rao, Y. and others (2011). Nicotine decreases food intake through activation of POMC neurons. Science, 332, 1330-1332.

Mokdad, A. H., Marks, J. S., Stroup, D.F., and Gerberding, J. L. (2004). Actual causes of death in the United States. The Journal of the American Medical Association, 291 (10), $1238-1245$.

Moran, S., Wechsler, H., Rigotti, N.A. (2004). Social smoking among US college students. Pediatrics, 114(4), 1028-1034.

Mytton, O. ,Clarke, D., Rayner, M. (2012) Taxing unhealthy food and drinks to improve health. British Medical Journal, 344:e2931.

Pacula, R. L. (1998). Does increasing the beer tax reduce marijuana consumption?Journal of Health Economics, 17(5),557-585. 
Philipson, T.J., R.A. Posner (2008). Is the obesity epidemic a public health problem? A review of Zoltan J. Acs and Alan Lyles's obesity, business and public policy. Journal of Economic Literature, 46, 974-982.

Rashad, I., Grossman, M. (2004). The economics of obesity. Public Interest, 156, 104-112.

Rashad, I., Grossman, M., Chou, S. (2006). The super size of America: An economic estimation of body mass index and obesity in adults. Eastern Economic Journal, 32, 133-148.

Schane, R. E., Glantz, S. A. and Ling, P. M. (2009). Nondaily and social smoking: an increasingly prevalent pattern. Archives of Internal Medicine, 169(19), 1742-1744.

Spring, B., Howe, D., Berendsen, M. and others (2009). Behavioral intervention to promote smoking cessation and prevent weight gain: a systematic review and meta-analysis, Addiction, 104(9),1472-1486.

Yaniv, G., Rosin, O., Tobol, Y. (2009). Junk-food, home cooking, physical activity and obesity: the effect of the fat tax and the thin subsidy. Journal of Public Economics, 93, 823-830.

Wehby, G. L., Courtemanche, C. J. (2012). The heterogeneity of the cigarette price effect on the body mass index. Journal of Health Economics, 31, 719-729.

WHO (2000). Obesity: preventing and managing the global epidemic. Report of a WHO Consultation. WHO Technical Report Series 894. World Health Organization, Geneva.

\section{A Appendix}

\section{A.1 The solution under bounded rationality}

Here we study the extreme case in which the agent does not take into account the future consequences of her current eating and smoking choices on future body weight and addiction to smoking. In other words, we consider the case where, at each time $t$, the agent maximizes (5) given the budget constraint, her current body weight and her current addiction to smoking, but without taking into account (6) and (7). This case is instructive, because it allows to determine the optimal solution of an agent that, due to informational or cognitive constraints, does not consider (or is not able to determine) the rational forward-looking path of choices that would maximize her intertemporal utility. The agent thus takes into account current addiction and body weight when choosing the optimal level of smoking and food consumption, but she ignores that future addiction and body weight will change as a consequence of her current choices. As shown in the main text, this naïve approach is not optimal for a forwardlooking agent, because it does not allow to take into full consideration how the future evolution of addiction and body weight will impact on future utility.

Given current body weight and addiction to smoking, the optimal choice of food con- 
sumption and smoking satisfies the following condition

$$
\frac{U_{c}}{U_{s}}=\frac{p^{c}}{p^{s}}
$$

which is formally equivalent to the familiar static optimization solution under budget constraint where the marginal rate of substitution between two goods equals the ratio of the corresponding market prices. However, here the solution is not static because the optimal amount of smoking and eating depends on addiction to smoking and body weight. Since addiction and body weight change as a consequence of the choices of the agent, they are going to affect $U_{c} / U_{s}$ and, consequently, the optimal level of food consumption and smoking that satisfies (16) will change over time. This process stops when (16) holds and $\dot{w}=\dot{a}=0$. Whether this condition is associated to being over- or underweight, or with being on diet or binging, requires additional specific assumptions. For a forward-looking agent, instead, we have shown that $U_{c} / U_{s}=p_{c} / p_{s}$ results in steady state if the agent has a healthy body weight and is not addicted to smoking.

\section{A.2 Proof of Proposition 1}

It is convenient to express the conditions (8)-(11) as a systems of differential equations where only control and state variables appear. Differentiating (8) and (9), replacing (10), (11), and using (8), (9), the following dynamic system results

$$
\begin{aligned}
\dot{s} & =\frac{1}{\psi}\left(A U_{c s}-B U_{c c}\right) \\
\dot{c} & =\frac{1}{\psi}\left(B U_{c s}-A U_{s s}\right) \\
\dot{a} & =s-\delta a \\
\dot{w} & =c-\varepsilon s-\delta w .
\end{aligned}
$$

where

$$
\begin{aligned}
A & =(\delta+\rho)\left(p^{c}-U_{c}\right)-U_{w}, \\
B & =(\delta+\rho)\left(p^{s}-U_{s}\right)-U_{a}+\dot{a} U_{s a}+\varepsilon U_{w}, \\
\psi & =U_{c c} U_{s s}-U_{c s}^{2}>0,
\end{aligned}
$$

In steady state, conditions (17)-20 must be equal to zero. Since $\psi>0$ holds by assumption, this implies the following:

$$
\begin{aligned}
U_{w} & =(\delta+\rho)\left(p^{c}-U_{c}\right) \\
U_{a} & =(\delta+\rho)\left(p^{s}-U_{s}\right)+\varepsilon U_{w} \\
\delta w^{s s} & =c^{s s}-\varepsilon s^{s s} \\
\delta a^{s s} & =s^{s s}
\end{aligned}
$$




\section{A.3 Asymptotic stability of the steady state}

At the steady state, the eigenvalues of the Jacobian matrix $J$ of the dynamic system (17)-(20) are (Dockner, 1985)

$$
e_{1,2,3,4}=\frac{\rho}{2} \pm \sqrt{\frac{\rho^{2}}{4}-\frac{K}{2} \pm \frac{1}{2} \sqrt{K^{2}-4|J|}}
$$

where $|J|$ is the determinant of the Jacobian,

$$
\begin{aligned}
|J|= & \frac{1}{\psi}\left[(2 \delta+\rho) U_{s a}+U_{a a}+\delta(\delta+\rho) U_{s s}\right]\left[\delta(\delta+\rho) U_{c c}+U_{w w}\right] \\
& -\frac{1}{\psi}\left[\delta^{2}(\delta+\rho)^{2} U_{c s}^{2}-\delta \varepsilon(\delta+\rho)\left(\varepsilon U_{c c}+2 U_{c s}\right) U_{w w}\right]
\end{aligned}
$$

and

$$
\begin{aligned}
K= & -2 \delta(\delta+\rho)-\frac{1}{\psi}\left[(2 \delta+\rho) U_{s a}+U_{a a}\right] U_{c c} \\
& -\frac{1}{\psi}\left[U_{s s}+\varepsilon\left(\varepsilon U_{c c}+2 U_{c s}\right)\right] U_{w w} .
\end{aligned}
$$

After some algebraic manipulations, it can be shown that $K^{2}>4|J|$. The conditions $|J|>0$ and $K<0$ are sufficient for saddle point stability (Dokner, 1985). The former condition requires

$$
U_{s a}<-\frac{\delta(\delta+\rho) U_{s s}+U_{a a}}{2 \delta+\rho}+\frac{\delta^{2}(\delta+\rho)^{2} U_{c s}^{2}-\delta \varepsilon(\delta+\rho)\left(\varepsilon U_{c c}+2 U_{c s}\right) U_{w w}}{(2 \delta+\rho)\left[\delta(\delta+\rho) U_{c c}+U_{w w}\right]}=\alpha_{1},
$$

the latter requires

$$
U_{s a}<\frac{2 \delta(\delta+\rho) \psi-\left[U_{s s}+\varepsilon\left(\varepsilon U_{c c}+2 U_{c s}\right)\right] U_{w w}-U_{a a} U_{c c}}{(2 \delta+\rho) U_{c c}}=\alpha_{2} .
$$

Hence stability of the steady state requires $U_{s a}<\min \left\{\alpha_{1}, \alpha_{2}\right\}$. Figure 2 is drawn for the case where $\alpha_{1}<\alpha_{2}$, i.e. stability is guaranteed when $|J|>0$.

\section{A.4 Increasing the price of smoking}

Short-run effect. For given values of the state and costate variables, the instantaneous reaction to a change in the price of smoking $p^{s}$ is obtained by applying the implicit function theorem to (8) and (9):

$$
\begin{aligned}
\frac{\partial s(t)^{*}}{\partial p^{s}} & =\frac{U_{c c}}{\psi}<0 \\
\frac{\partial c(t)^{*}}{\partial p^{s}} & =\frac{U_{c s}}{\psi} .
\end{aligned}
$$

This implies that, in the short-run, smoking decreases, while food intake increases if $U_{c s}>0$ and it decreases otherwise. 
Long-run effect. The change in the steady state demand for smoking as a response to a change in the price of smoking is given by the following expression:

$$
\frac{\partial s^{s s}}{\partial p^{s}}=-\frac{|P|}{|J|}
$$

where $P$ is

$$
P=\left[\begin{array}{llll}
\partial \dot{s} / \partial p^{s} & \partial \dot{s} / \partial c & \partial \dot{s} / \partial a & \partial \dot{s} / \partial w \\
\partial \dot{c} / \partial p^{s} & \partial \dot{c} / \partial c & \partial \dot{c} / \partial a & \partial \dot{c} / \partial w \\
\partial \dot{a} / \partial p^{s} & \partial \dot{a} / \partial c & \partial \dot{a} / \partial a & \partial \dot{a} / \partial w \\
\partial \dot{w} / \partial p^{s} & \partial \dot{w} / \partial c & \partial \dot{w} / \partial a & \partial \dot{w} / \partial w
\end{array}\right]
$$

Since the following holds

$$
|P|=-\delta(\delta+\rho) \frac{\delta(\delta+\rho) U_{c c}+U_{w w}}{\psi}>0 .
$$

and a necessary condition for a stable steady state is that the determinant of the Jacobian matrix is positive, then

$$
\frac{\partial s^{s s}}{\partial p^{s}}=\delta(\delta+\rho) \frac{\delta(\delta+\rho) U_{c c}+U_{w w}}{\psi|J|}<0
$$

Similarly, we can compute the change in steady state food consumption and body weight and obtain:

$$
\begin{aligned}
\frac{\partial c^{s s}}{\partial p^{s}} & =\delta(\delta+\rho) \frac{\varepsilon U_{w w}-\delta(\delta+\rho) U_{c s}}{\psi|J|} \\
\frac{\partial w^{s s}}{\partial p^{s}} & =-\delta(\delta+\rho)^{2} \frac{\varepsilon U_{c c}+U_{c s}}{\psi|J|}
\end{aligned}
$$

This implies the following:

$$
\begin{gathered}
\frac{\partial c^{s s}}{\partial p^{s}}>0 \quad \Leftrightarrow \quad U_{c s}<\sigma_{1} \\
\frac{\partial w^{s s}}{\partial p^{s}}>0 \quad \Leftrightarrow \quad U_{c s}<\sigma_{2},
\end{gathered}
$$

where

$$
\begin{aligned}
\sigma_{1} & =\frac{\varepsilon}{\delta(\delta+\rho)} U_{w w} \leq 0, \\
\sigma_{2} & =-\varepsilon U_{c c} \geq 0 .
\end{aligned}
$$




\section{A.5 Increasing the price of food}

Short-run effect. To obtain the instantaneous reaction to a change in the price of food, we apply the implicit function theorem to (8) and (9):

$$
\begin{aligned}
\frac{\partial c(t)^{*}}{\partial p^{c}} & =\frac{U_{s s}}{\psi}<0 ; \\
\frac{\partial s(t)^{*}}{\partial p^{c}} & =\frac{U_{c s}}{\psi} .
\end{aligned}
$$

This implies that, in the short-run, food consumption decreases, while smoking increases if $U_{c s}>0$ and it decreases otherwise.

Long-run effect. In the long-run, the impact of a permanent change in the price of consumption on food consumption, body weight and smoking is the following:

$$
\begin{aligned}
\frac{\partial c^{s s}}{\partial p^{c}} & =\delta(\delta+\rho) \frac{(2 \delta+\rho) U_{s a}+U_{a a}+\delta(\delta+\rho) U_{s s}+\varepsilon^{2} U_{w w}}{\psi|J|} \\
\frac{\partial w^{s s}}{\partial p^{c}} & =(\delta+\rho) \frac{(2 \delta+\rho) U_{s a}+U_{a a}+\delta(\delta+\rho) U_{s s}+\delta \varepsilon(\delta+\rho) U_{c s}}{\psi|J|} \\
\frac{\partial s^{s s}}{\partial p^{c}} & =\delta(\delta+\rho) \frac{\varepsilon U_{w w}-\delta(\delta+\rho) U_{c s}}{\psi|J|}
\end{aligned}
$$

It can be shown that $\partial c^{s s} / \partial p^{c}$ is always negative when the concavity condition on the utility function and the necessary condition for stability $(|J|>0)$ hold. The same is not true for $\partial w^{s s} / \partial p^{c}$. When the metabolic effect is non negligible, $\varepsilon>0$, condition (27) implies:

$$
\frac{\partial w^{s s}}{\partial p^{c}}>0 \quad \Leftrightarrow \quad U_{c s}>\sigma_{3}
$$

where

$$
\sigma_{3}=-\frac{(2 \delta+\rho) U_{s a}+U_{a a}+\delta(\delta+\rho) U_{s s}}{\delta(\delta+\rho) \varepsilon} .
$$

In passing by, note that $\sigma_{3}=0$ is the bifurcation condition for instability of the steady state in the Becker and Murphy (1988) model. In the special case where there is no metabolic effect of smoking $(\varepsilon=0)$, then $\partial w^{s s} / \partial p^{c}=\left(\partial c^{s s} / \partial p^{c}\right) / \delta<0$. Finally, condition 28) implies

$$
\frac{\partial s^{s s}}{\partial p^{c}}>0 \quad \Leftrightarrow \quad U_{c s}<\sigma_{1}
$$




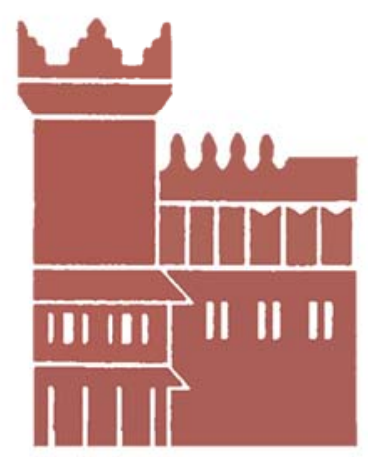

Alma Mater Studiorum - Università di Bologna DEPARTMENT OF ECONOMICS

Strada Maggiore 45

40125 Bologna - Italy

Tel. +39051 2092604

Fax +390512092664

http://www.dse.unibo.it 